\title{
sciendo
}

Current Issues in Pharmacy and Medical Sciences

Formerly ANNALES UNIVERSITATIS MARIAE CURIE-SKLODOWSKA, SECTIO DDD, PHARMACIA

journal homepage: http://www.curipms.umlub.pl/

\section{Methods of isolation and bioactivity of alkaloids obtained from selected species belonging to the Amaryllidaceae and Lycopodiaceae families}

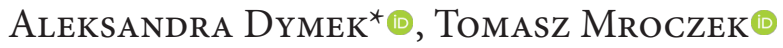 \\ Independent Laboratory of Chemistry of Natural Products, The Chair of Pharmacognosy, Medical University of Lublin, Poland
}

\begin{tabular}{|c|c|}
\hline ARTICLE INFO & ABSTRACT \\
\hline $\begin{array}{l}\text { Received } 17 \text { February } 2021 \\
\text { Accepted } 20 \text { May } 2021\end{array}$ & $\begin{array}{l}\text { Alkaloids obtained from plants belonging to the Amaryllidaceae and Lycopodiaceae } \\
\text { families are of great interest due to their numerous properties. They play a very important }\end{array}$ \\
\hline $\begin{array}{l}\text { Keywords: } \\
\text { Lycopodium sp., } \\
\text { Narcissus sp., } \\
\text { AChE inhibitors, } \\
\text { TLC, } \\
\text { SPE, } \\
\text { PLE, } \\
\text { TLC-bioatography. }\end{array}$ & $\begin{array}{l}\text { role mainly due to their strong antioxidant, anxiolytic and anticholinesterase activities. } \\
\text { The bioactive compounds obtained from these two families, especially galanthamine } \\
\text { and huperzine A, have found application in the treatment of the common and } \\
\text { incurable dementia-like Alzheimer's disease. Thanks to this discovery, there has been } \\
\text { a breakthrough in its treatment by significantly improving the patient's quality of life and } \\
\text { slowing down disease symptoms - albeit with no chance of a complete cure. Therefore, } \\
\text { a continuous search for new compounds with potent anti-AChE activity is needed } \\
\text { in modern medicine. In obtaining new therapeutic bioactive phytochemicals from plant } \\
\text { material, the isolation process and its efficiency are crucial. Many techniques are known } \\
\text { for isolating bioactive compounds and determining their amounts in complex samples. } \\
\text { The most commonly utilized methods are extraction using different variants of organic } \\
\text { solvents allied with chromatographic and spectrometric techniques. Optimization } \\
\text { of these methods and modification of their procedures potentially allows researchers } \\
\text { to obtain the expected results. The aim of this paper is to present known techniques } \\
\text { for the isolation of alkaloids, especially from three species Narcissus, Lycopodium and } \\
\text { Huperzia that are a rich source of AChE inhibitors. In addition, innovative combinations } \\
\text { of chromatographic and spectrometric methods and novel TLC-bioautography will be } \\
\text { presented to enable researchers to better study the bioactivity of alkaloids. }\end{array}$ \\
\hline
\end{tabular}

\section{INTRODUCTION}

Plants belonging to the Amaryllidaceae and Lycopodiaceae families are a rich source of bioactive alkaloids. These compounds show promising pharmacological activities, including anti-inflammatory, anxiolytic and antioxidant activity. Such alkaloids demonstrate strong neuroprotective and antidepressant properties, and, due to their ability to inhibit acetylcholinesterase (AChE) activity, they have been used in the treatment of neurodegenerative illnesses. This property was helpful in treating Alzheimer's disease which is the most common cause of dementia worldwide. The etiology of this disease is still unclear. The neurodegenerative disease process is characterized by acetylcholine (ACh) decrease in the cholinergic neurons and damaged brain structures and nerve cells in the central nervous system

\footnotetext{
* Corresponding author

e-mail: aleksandra.dymek91@interia.pl
}

(CNS) [1,2]. Unfortunately, it is an age-related progressive and incurable disorder, and current treatment methods are not satisfactory. The therapy consists only in increasing the ACh concentration at the synaptic site in the brain and suppressing the disease symptoms characterized by the deterioration of memory leading to remarkable cognitive disorders. By improving neurotransmission in the cholinergic system, it alleviates behavioral disorders, improves memory and the patient's quality of life $[3,4]$.

Cholinesterase inhibitors are the only available first line pharmacotherapy used to treat patients suffering from mild to moderate Alzheimer's disease. Their action is to effectively limit the breakdown of the neurotransmitter ACh by inhibiting the ACh-hydrolyzing enzyme. One of these, galathamine, which is marketed as Nivalin ${ }^{\circ}$, was isolated for the first time from the snowdrop Galanthus species belonging to the Amaryllidaceae family. This is not 
the only example of a compound showing potent $\mathrm{AChE}$ inhibitory activity [5,6]. Huperzine A (HupA), isolated from the Huperzia species belonging to the Lycopodiaceae family, shows surprising results in that it holds the ability to reversibly and selectively inhibit the activity of AChE. Moreover, this alkaloid has an antioxidant activity against free radicals and $\beta$-amyloid - both responsible for cellular toxicity. On the basis of many studies, huperzine $\mathrm{A}$ proved to have better penetration through the blood-brain barrier and longer duration of AChE inhibitory action in contrast to available drugs, and trials have led to HupA being proposed as a potential drug for the treatment of Alzheimer's disease. High concentrations of this alkaloid are found in the Huperzia species, one of the most popular natural plants in the Lycopodiaceae family.

The growing interest in galanthamine and HupA has very quickly led to an increased risk of extinction for these species and thus a reduction in the resources of these substances $[7,8]$. Therefore, many researchers have been investigating the phytochemical constituents in the Amaryllidaceae and Lycopodiaceae families. Numerous studies have allowed researchers to detect new alkaloids with similar properties and strong anti-AChE activity. Many techniques have been used to isolate AChE inhibitors. Moreover, many attempts have been made to combine several methods to better isolate, separate and obtain relatively pure fractions of AChE inhibitors [9-11].

In view of the fact that species of the genus Lycopodium, Narcissus and Huperzia belonging to the aforementioned families have a lot to offer in various areas of science, in our work, we will present well known techniques for the isolation of AChE inhibitors. The aim of this review is also to present the novel technique of TLC-bioautography as a rapid and relatively inexpensive assay to prove the ability of active components (inhibitors) to inhibit selected enzymatic activities. A review of the techniques presented provides a basis for the efficient isolation of potent AChE inhibitors, which may be crucial for the treatment of neurodegenerative diseases in the 21 st century.

\section{METHODS OF ISOLATION}

The alkaloids of the Lycopodium, Huperzia and Narcissus species constitute a numerous, heterogeneous group of compounds whose isolation can cause many difficulties. Since most of them have a heterocyclic nitrogen atom in their structure and show alkaline properties, the isolation of the constituents from plant material is carried out using extraction with an organic solvent. The basis of most chemical isolation and identification processes are separation techniques, which involve a selective fractionation of a chemical substance. The extraction process itself is a method of extricating individual substances by dissolving them in an appropriately selected solvent and separating them from the remaining insoluble components of the sample. The extracted substance is separated between two immiscible phases; therefore the correct choice of a solvent is an extremely important step in ensuring optimal conditions for the isolation process $[12,13]$. However, despite such a frequent use and interest in these compounds, there is not enough data on methods for their extraction and isolation.

To date, researchers have only used conventional methods to obtain the bioactive alkaloids of Lycopodiaceae and Amaryllidaceae. However, a number of papers have been published using different organic solvents such as methanol, ethanol, hexane, chloroform and many others at various concentrations $[14,15]$. The application of these solvents has led to the isolation of alkaloids and confirmation of many of their properties, including anti-AChE activity. The isolation of the major Lycopodiaceae alkaloids was carried out by Konrath [10]. Dried and ground aerial parts from two Lycopodium species were extracted using the Soxhlet apparatus and maceration with n-hexane as a solvent. The yield of these two extracts was very low and did not exceed $0.4 \%$. However, it allowed the researcher to obtain the main Lycopodium alkaloids such as lycopodine, lycodine or $\alpha$-obscurine [10].

Another research team obtained higher extraction efficiency. Orhan et al. [16] tested four Lycopodium extracts obtained by maceration using four different organic solvents: petroleum ether, chloroform, ethyl acetate and methanol. In vivo studies confirmed the high efficiency of Lycopodiaceae samples, especially chloroform extracts, which displayed potent anti-inflammatory activity [16]. Still, alternatives to these methods were sought because they required huge amounts of solvents, generated large amounts of waste, and were time-consuming. The low efficiencies of the extracts obtained and the low recovery of alkaloids led researchers to optimize known methods and to develop new, more efficient ones. For example, Da Silva and co-authors [17] applied a modern extraction method. They utilised supercritical fluid extraction (SFE) to a high quantity of the alkaloid lycopodine from Lycopodium clavatum $\mathrm{L}$.

As a result of many studies, Da Silva concluded that the optimization of extraction conditions such as temperature and pressure increases are significant and gave a satisfactory efficiency of lycopodine above $20 \%$. The method developed by Da Silva et al. has been widely reported in the analytical literature as an effective way to optimize many processes, however, many researchers have attempted to combine several methods to better isolate bioactive compounds. To this end, Zhang et al. [18] used a combination of several techniques: an ultrasonic assisted method, macroporous resin column chromatography and liquid-liquid extraction to isolate and purify the potent anti-AChE inhibitors HupA and huperzine B (HupB) from Huperzia species. The combination and optimization of these methods ultimately led to the isolation of a high yield of these alkaloids [17-19].

The isolation and identification of this alkaloid was also carried out using an extraction with different solvents and chromatographic methods coupled with mass spectrometry (MS). For the first time, Qingqing and Yuenha reported the analysis of alkaloid compounds from Huperzia serrata using high performance liquid chromatography coupled with a diode array detector and tandem mass spectrometry techniques (HPLC-DAD-ESI-MS-MS). Thanks to these coupled methods, the alkaloids compounds in the extract could be identified successfully in a single run [20]. 
The best method to isolate HupA, as well as other new potent $\mathrm{AChE}$ inhibitors is still being sought. Currently, this alkaloid and its numerous analogues are obtained by chemical synthesis. However, due to the high cost of this process, extraction from plants belonging to the Lycopodiaceae family is still the main source of HupA.

Because of the tremendous interest in the Huperzia species and the awareness that it is a good source of potent HupA, it has become an endangered species. Therefore, researchers sometimes have to settle for a small amount of plant materials to conduct analytical studies. Interesting results are described by Cuthbertson [21], who used an improved method for microscale extraction of HupA from the clubmoss Huperzia squarrosa. With just $3 \mathrm{mg}$ of plant material, the recovery of HupA was as high as $99.95 \%$. The use of this methodology could potentially contribute to the isolation of a potent $\mathrm{AChE}$ inhibitor, but also to the conservation of valuable plant resources [21]. However, attempts to find new inhibitors that may prove to be as potent as HupA are worthwhile.

Before we describe the isolation methods of Narcissus species, it is worth mentioning the isolation of galanthamine, which has led to a giant breakthrough in the treatment of Alzheimer's disease. This alkaloid is a natural product known from several members belonging to the Amaryllidaceae family. It was isolated for the first time from the leaves and flowers of the snowdrop (Galanthus woronowii) $[5,6]$. Today, it is obtained from Narcissus and Leucojum species, as well as synthetically. For the extraction of alkaloids from the Amaryllidaceae family, standard methods are used. These include percolation and Soxhlet's extraction. To the best of our knowledge, several works using methanol or ethanol maceration with solvents at different temperatures have been published [22]. However, these are very time-consuming studies with possible drawbacks of artifact creation.

Plant material is usually processed with various types of organic solvents, and then purified chromatographically. Next, the chemical structures are increasingly elucidated by MS and 1D and 2D NMR spectroscopic analyses. Such an approach was presented by Safratova [23] whose study allowed her to isolate as many as 15 alkaloids of which two had been unidentified so far [23]. In turn, Borra et al. [24] developed and optimized an alternative protocol for the isolation of narciclasine from Amaryllidaceae bulbs. This approach was based on a specific solvent partitioning sequence that allowed the separation of narciclasine from other constituents and to yield a trace of 2-epi-narciclasine for the first time [24]. New reports on other more modern techniques for alkaloid extraction from Amaryllidaceae are still scarce. Researchers have conducted isolation trials utilising: microwave-assisted extraction (MAE) using methanol and 450W microwave power, ultrasound-assisted extraction (USAE) with methanol at $25^{\circ} \mathrm{C}$ or supercritical fluid extraction (SFE). These methods were not optimized, so the alkaloid recovery was low and the duration of the procedures was still too long [22].

In our laboratory, a modern pressurized liquid extraction (PLE) was successfully applied to obtain alkaloid extracts. For this purpose, different solvents and solutions of diverse polarity were used. The crude extracts were then purified by solid phase extraction (SPE) on special cartridges. This method is relatively fast and allows the obtaining of preliminary purification of samples from excess ballast substance. Thus, the Amaryllidaceae alkaloids (such as galanthamine or lycorine) were eluted with $80-100 \%$ recovery [22]. The same procedure was used for the extraction and purification of Lycopodiaceae alkaloids [25]. These methods allowed gaining results with high accuracy and ascertaining the influence of types of solvents on the obtained amount of alkaloids. Another purification method was employed with appropriate combinations of sorbents and a vacuum to increase the flow rate. This method was innovatively carried out using gradient vacuum liquid chromatography (gVLC) with two sorbents in various proportions packed in polypropylene cartridges. The preparation of the columns did not require too much work and time, and the sorbents used proved to be suitable for the initial purification of plant fractions. This allowed us to minimize the analysis time, by omitting some of analytical processes. The next stage included the identification of alkaloids by HPLC/ ESI-QTOF-MS in order to compare which combination of sorbents would allow us to obtain the highest concentration of AChE inhibitors. These optimized and coupled techniques have become a useful tool for the identification of many Narcissus alkaloids [22,26].

\section{BIOACTIVITY METHODS}

Once a properly selected extraction and isolation method has been carried out, it is time to assess the activity of the obtained compounds. Bioactivity methods are widely used to detect many important properties of plant materials, such as antifungal, antimicrobial, radical scavenging and antioxidant activities. Literature data have revealed a clear trend towards using these techniques to detect enzyme or inhibitor activity.

Several analytical procedures are currently applied for screening $\mathrm{AChE}$ inhibitors from plant materials, and HPLC and GC methods with MS detection are widely used. However, their drawbacks are insufficient sensitivity, mass accuracy and separation efficiency. Moreover, some unstable alkaloids cannot be analyzed in this way [22,27].

TLC-bioautography is a very convenient and innovative method for confirming AChE inhibitory activity by alkaloids. It has proven to be an effective method for detecting compounds with inhibitory activity that is compatible with TLC-plates. According to Marston, the term 'bioautography' refers to experiments performed to detect the growth of a microorganism on a TLC plate, while the term 'autography' refers to the use of chemical methods to identify biological processes such as detection of enzyme inhibitory activity from plant extracts. TLC-bioautography has been successfully used for the screening of inhibitors of many enzymes, for example, AChE, butyrylocholinesterase, dipeptidyl peptidase IV, glucose-6-phosphate dehydrogenase, tyrosinase and many others $[27,28]$. Since it was discovered that some alkaloids of the Amaryllidaceae and Lycopodiaceae families (including galanthamine or HupA) are potent cholinesterase inhibitors, Narcissus, Lycopodium and Huperzia extracts have become of interest for testing by TLC-bioautography. 
To date, several approaches to TLC-bioautography are known. One, based on Ellman's method has been employed to study AChE inhibition in several works [22,29]. However, it has been replaced by another method due to false positive effects and an unstable yellow background. An alternative 1-naphtyl acetate method was, therefore, applied as a substrate of AChE. The resulting $\alpha$ - naphtyl reacts with Fast Blue B Salt to give a purple background. In this method, the presence $\mathrm{AChE}$ inhibitors are identified as white bands. However, it was necessary to improve the sensitivity of this method.

A very good solution to this problem, proposed by Prof. Tomasz Mroczek, was the employment of 2-naphtyl acetate as a substrate of the enzyme, and the addition of this substrate to the mobile phase allowed the obtaining of more concise and stable inhibition zones on the TLC plates. In this enhanced method, 2-naphtyl acetate is converted to 2-naphtol by the enzyme. The deep purple background of TLC-plates was obtained by reacting 2-naphtol with Fast Blue B Salt reagent to visualize zones and inhibitors. The condensation reaction quickly resulted in a deep purple azo dye. Importantly, white AChE inhibitors zones on the deep purple background were visible already after a period of $1 \mathrm{~min}$ and were stable for at least one day [27,28,30-34].

This improved TLC-bioautography was used in screening known and new potent $\mathrm{AChE}$ inhibitors in Narcissus purified extracts and in individual alkaloid fractions (Fig.1). This method is described in detail in some published papers. These studies show that TLC-bioautography can be successfully combined with other separation and identification techniques. The combination of TLC-bioautography, HPTLC-densitometry and HPLC/ESI-TOF-MS methods, for instance, allows the determination of the peak of dihydrogalanthamine, which exhibits more potent AChE inhibitory activity than the better-known galanthamine. In addition, a combination of extraction technique and application of the pioneering gVLC approach combined with TLC-bioautography has yielded visualizations with inhibitory white spots that are likely to be the potent $\mathrm{AChE}$ inhibitors lycorine, ungeremine and sanguinine. These are naturally present in large percentages in Narcissus c.v. 'Hawera' species. Thus, this relatively novel technique gives quick access to information concerning the activity and the localisation of the activity in a complex plant matrix. Thanks to this method, it is possible to rapidly and efficiently detect many AChE inhibitors from plant substances [22,26,34].

Currently, in our laboratory at the Medical University of Lublin, research is being conducted to seek new AChE inhibitors in species belonging to the Amaryllidaceae and Lycopodiaceae families. Interestingly, the analysis is being done using a modern and automated device known to be capable of TLC derivatization (Fig. 2). It ensures reproducible results by using a unique "micro-droplet" spraying technology. The derivatizer provides homogeneity and convenience in reagent application, which proves to be better than manual spraying. As a result, the total analysis time is reduced. Moreover, the usage of expensive enzyme is reduced twofold. In addition, this method is safe for the analyst and the environment due to closed system processing. Because of the different physicochemical properties of the reagents used (such as viscosity), several spray nozzles are available. This optimized method for confirming the activities of many compounds such as AChE inhibitors is significant in many scientific fields. Already it is stating to play a key role in the treatment of Alzheimer's disease, by finding pharmaceutical agents suitable not only in alleviating the symptoms of the disease, but also in attempts at causal treatment.

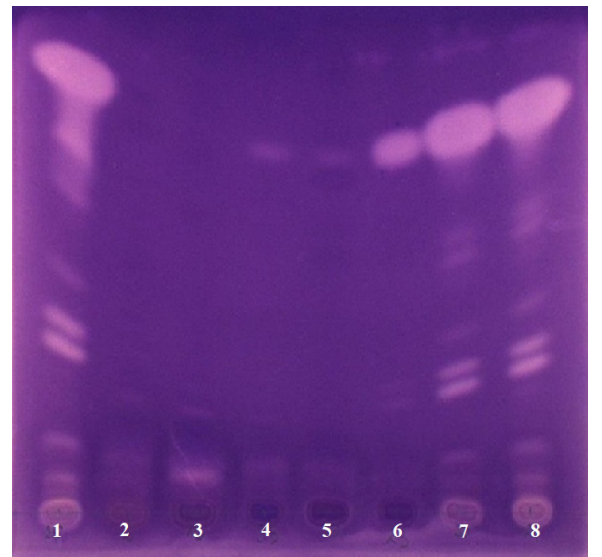

Figure 1. Videoscan of TLC with bioauthography - the application of the modified 'Fast Blue B reagent' method. Here, the presence of potent AChE inhibitors is indicated by white inhibition zones on a deep purple background. The tracks follow fractions obtained from Narcissus triandrus L. c.v. 'Hawera' using PLE and VLC

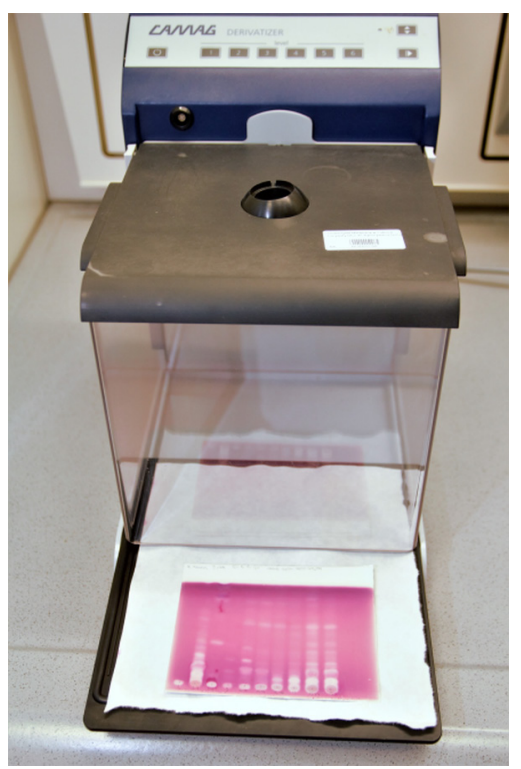

Figure 2. Automated spraying device for reagent transfer onto TLC plates

\section{CONCLUSIONS}

To date, a number of structurally diverse alkaloids have been isolated and identified from plants of the Amaryllidaceae and Lycopodiaceae families. Taking into account the richness of bioactive compounds present in plant materials and their wide application, it is expected that more new alkaloids will be isolated in the future. Isolation of alkaloids from plant materials is one of the most important steps at the beginning of an experimental path. Every subsequent process (sample purification, identification of alkaloids or 
TLC- bioautography of active AChE inhibitors) depends on this step. The analytical techniques described in this paper clearly demonstrate progress in developing procedures for the separation and isolation of Lycopodiaceae and Amaryllidaceae alkaloids. Once-standard techniques have now been successfully replaced by those utilizing sophisticated apparatuses that allow researchers to rapidly separate sample components, regardless of aggregate state. Improving the extraction conditions by optimizing the method and combining it with innovative sample purification e.g. by VLC or SPE, allows researchers to obtain extracts with high alkaloid recoveries. Their combination with chromatographic and spectroscopic approaches enables scientists not only to isolate and to determine potent anti-AChE inhibitors, but also to prepare and purify many substances, including drugs of plant origin.

Bioautography with TLC is a significant extension of current procedures for the determining of AChE inhibitors in extracts. This is due to the simplicity, low cost and reproducibility of this method. The potential for an increased application of bioactivity methods for selective and sensitive detection of this technique is huge. Although many bioassays have already been successfully performed, there are many opportunities to increase the number of bioautographic methods, especially for enzymes.

The detection of galanthamine and its subsequent marketing as a selective AChE inhibitor for the treatment of Alzheimer's disease was a major breakthrough in medicine. This has led to further discoveries, such as the isocarbostyril alkaloids of Amaryllidaceae, which are in preclinical development as very promising anticancer agents [35]. This approach gives hope in the treatment of many disease entities, including lifestyle diseases.

According to current WHO (World Health Organization) data, neurodegenerative disorders are a rapidly growing problem, both medically and socially. The number of patients with Alzheimer's disease is expected to rise as life expectancy increases and the population ages. The treatment of neurodegenerative diseases continues to be a focus for researchers. Efforts are constantly being made to find new ways to treat and prevent these disorders. Although research on the biosynthesis of Amaryllidaceae alkaloids began as early as the 1960s, their biosynthetic pathways are far away from being fully understood. Interestingly, modern medicine, disappointed with synthetic medicine, is more and more reaching out to Nature and the richness of the plant world to find antidotes for many diseases. Therefore, the search for natural AChE inhibitors is a cutting edge approach to drug discovery and to disease treatment.

\section{CONFLICT OF INTEREST}

The authors have no conflict of interest to declare.

\section{ORCID iDs}

Aleksandra Dymek (Dhttps://orcid.org/0000-0002-5285-760X Tomasz Mroczek (Dhttps://orcid.org/0000-0002-5267-7182

\section{REFERNCES}

1. Chaves SKM, Feitosa CM, da S. Araujo L. Alkaloids pharmacological activities - prospects for the development of phytopharmaceuticals for neurodegenerative diseases. Curr Pharm Biotechnol. 2016;17(7):629-95.

2. Weller J, Budson A. Current understanding of Alzheimer's disease diagnosis and treatment. F1000Res. 2018;7:F1000.

3. Zabłocka A. Choroba Alzheimera jako przykład schorzenia neurodegeneracyjnego. Postepy Hig Med Dosw. 2006;60:209-16.

4. Cummings JL, Vinters HV, Cole GM, Khachaturian ZS. Alzheimer's disease: etiologies, pathophysiology, cognitive reserve, and treatment opportunities. Neurology. 1998;51:2-17.

5. Russo P, Frustaci A, Fini M, Cesario A. From traditional European medicine to discovery of new drug candidates for the treatment of dementia and Alzheimer's disease: Acetylcholinesterase inhibitors. Curr Med Chem. 2013;20:976-83.

6. Heinrich M, HL. Galanthamine from snowdrop - the development of a modern drug against Alzheimer's disease from local Caucasian knowledge. J Ethnopharmacol. 2004;92:147-62.

7. Ferreira A, Rodrigues M, Fortuna A, Falcao A, Alves G. Huperzine A from Huperzia serrata: a review of its sources, chemistry, pharmacology and toxicology. Phytochem Rev. 2016;15:51-85.

8. Zangara A. The psychopharmacology of huperzine A an alkaloid with cognitive enhancing and neuroprotective properties of interest in the treatment of Alzheimer's disease. Pharmacol Biochem Behav. 2003;75:675-86.

9. Li X, Kang M, Ma N, Pang T, Zhang Y, Jin H. et al. Identification and analysis of chemical constituents and rat serum metabolites in Lycopodium clavatum using UPLC-Q-TOF/MS combined with multiple data-processing approaches. Evid Based Complement Alternat Med. 2019;5165029:1-8.

10. Konrath EL, Neves BM, Lunardi PS, Passos CS, Simoes-Pires A, Ortega MG. et al. Investigation of the in vitro and ex vivo acetylcholinesterase and antioxidant activities of traditionally used Lycopodium species from South America on alkaloid extracts. J Ethnopharmacol. 2012;139:58-67.

11. Lopez S, Bastida J, Viladomat F, Codina C. Acetylcholinesterase inhibitory activity of some Amaryllidaceae alkaloids and Narcissus extracts. Life Sci. 2002;71:2521-9.

12. Ziegler J, Facchini PJ. Alkaloid biosynthesis: metabolism and trafficking. Annu Rev Plant Biol. 2008;59:735-69.

13. Jones WP, Kinghorn AD. Extraction of plant secondary metabolites. Methods Mol Biol. 2012;864:341-66.

14. Wu J, Wang H, Ma Y, Jiang J, Zhan R, Chen Y. Isolation of a new lycodine alkaloid from Lycopodium japonicum. Nat Prod Res. 2015;29(8):735-8

15. Tang Y, Li N, Zou Y, Ai Y, Ma G-L, Osman EEA et al. LC-MS guided isolation and dereplication of Lycopodium alkaloids from Lycopodium cernuum var. sikkimense of different geographical origins. Phytochemistry. 2019;160:25-30.

16. Orhan I, Kupeli E, Sener B, Yesilada E. Appraisal of antiinflammatory potential of the clubmoss, Lycopodium clavatum $\mathrm{L}$. J Ethnopharmacol. 2007;109:146-50.

17. Da Silva GF, Gandolfi PHK, Almeida RN, Lucas AM, Cassel E, Vargas RMF. Analysis of supercritical fluid extraction of lycopodine using response surface methodology and process mathematical modeling. Chem Eng Res Des. 2015;100:353-61.

18. Zhang H, Liang H, Kuang P, Yuan Q, Wang Y. Simultaneously preparative purification of Huperzine A and Huperzine B from Huperzia serrata by macroporous resin and preparative high performance liquid chromatography. J Chromatogr B. 2012; 904:65-72.

19. Kohler M, Haerdi W, Christen P, Veuthey J-L. Extraction of artemisinin and artemisinic acid from Artemisia annua L. using supercritical carbon dioxide. J Chromatogr A. 1997;785:353-60.

20. Wu Q, Gu Y. Quantification of huperzine A in Huperzia serrata by HPLC-UV and identification of the major constituents in its alkaloid extracts by HPLC-DAD-MS-MS. J Pharm Biomed Anal. 2006;40(4):993-8. 
21. Cuthbertson D, Piljac-Žegarac J, Lange BM. Validation of a microscale extraction and high-throughput UHPLC-QTOF-MS analysis method for huperzine A in Huperzia. Biomed Chromatogr. 2012;26(10)1191-5.

22. Mroczek T, Mazurek J. Pressurized liquid extraction and anticholinesterase activity-based thin-layer chromatography with bioautography of Amaryllidaceae alkaloids. Anal Chim Acta. 2009; 633:188-196.

23. Safratova M, Hostalkova A, Hulcova D, Breiterova K, Hrabcova V, Machado M. et al. Alkaloids from Narcissus poeticus cv. Pink Parasol of various structural types and their biological activity. Arch Pharm Res. 2018;41(2):208-18.

24. Borra S, Lapinskaite R, Kempthorne C, Liscombe D, McNulty J, Hudlicky T. Isolation, Synthesis, and Semisynthesis of Amaryllidaceae Constituents from Narcissus and Galanthus sp.: De Novo Total Synthesis of 2- epi-Narciclasine. J Nat Prod. 2018;81(6): 1451-9.

25. Dymek A, Widelski J, Wojtanowski KK, Płoszaj P, Zhuravchak $\mathrm{R}$, Mroczek T. Optimization of pressurized liquid extraction of Lycopodiaceae alkaloids from two Lycopodium species. Molecules. 2021;26:1626.

26. Mroczek T, Dymek A, Widelski J, Wojtanowski KK. The bioassayguided fractionation and identification of potent acetylcholinesterase inhibitors form Narcissus c.v. 'Hawera' using optimized vacuum liquid chromatography, high resolution mass spectrometry and bioautography. Metabolites. 2020;10:1-16.

27. Paradowska K, Polak B, Chomicki A, Ginalska G. Establishment of an effective TLC bioautographic method for the detection of Mycobacterium tuberculosis H37Ra phosphoglucose isomerase inhibition by phosphoenolpyruvate. J Enzyme Inhibit Med Chem. 2016;31(6):1712-7.
28. Paradowska K, Ginalska G. Zastosowanie metody TLC bioautografii do identyfikacji inhibitorów enzymatycznych. Tygiel. 2016;287-312.

29. Shetab-Boushehri SV. Ellman's method is still an appropriate method for measurement of cholinesterases activities. EXCLI J. 2018;17:798-9.

30. Mroczek T. Highly efficient, selective and sensitive molecular screening of acetylcholinesterase inhibitors of natural origin by solid-phase extraction-liquid chromatography/electrospray ionisation-octopole-orthogonal acceleration time-of-flightmass spectrometry and novel thin-layer chromatography-based bioautography. J Chromatogr A. 2009;12:2519-28.

31. Choma IM, Jesionek W. TLC-Direct Bioautography as a High Throughput Method for Detection of Antimicrobials in Plants. Chromatography. 2015;2(2):225-38.

32. Marston A, Kissling J, Hostettmann K. A rapid TLC bioautographic method for the detection of acetylcholinesterase and butyrylcholinesterase inhibitors in plants. Phytochem Anal. 2002;13(1):51-4.

33. Marston A. Thin-layer chromatography with biological detection in phytochemistry. J Chromatogr A. 2011;1218(19):2676-83.

34. Mroczek T. Qualitative and quantitative two-dimensional thin-layer chromatography/high performance liquid chromatography/diodearray/electrospray-ionization-time-of-flight mass spectrometry of cholinesterase inhibitors. J Pharm Biomed Anal. 2016;129:155-62.

35. Furst R. Narciclasine - an Amaryllidaceae alkaloid with potent antitumor and anti-Inflammatory properties. Planta Medica. 2016;82(16):1389-94. 